\title{
Wireless Transmission in Ventilation (HVAC) Ducts for the Internet of Things and Smarter Buildings: Proof of Concept and Specific Antenna Design
}

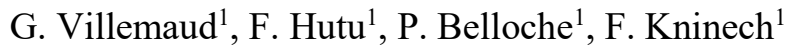 \\ guillaume.villemaud@insa-lyon.fr \\ ${ }^{1}$ Univ Lyon, INSA Lyon, Inria, CITI, F-69621 Villeurbanne, France
}

\begin{abstract}
We present here a preliminary study of wireless transmissions using the ventilation metallic ducts as waveguides. Starting from the waveguide theory, we deeply study in simulation the actual attenuation encountered by radiowaves in such a specific medium. This kind of wireless link appears to be really efficient, and therefore highly promising to implement Internet of Things (IoT) in old buildings to make them smarter. This paper also expresses a very simple empirical model in order to ease dimensioning a wireless network in such conditions and a specific antenna design enabling both good performance and high robustness to the influence of the environment.
\end{abstract}

Index Terms-HVAC, waveguide, IoT, empirical model.

\section{INTRODUCTION}

In order to increase the energy efficiency of large buildings, lots of efforts are dedicated to conceive Smart Buildings. But for buildings already constructed or even old buildings, this smartness could not be so simple to implement, as there are not smart-by-design. Here appears the concept of Smarter Buildings, where we have to find some simple ways of transforming old buildings in Smarter Buildings by adding some intelligence capabilities. But for those buildings to be smarter, they do not only need to have processing resources, but also lots of sensors to capture the relevant parameters to optimize the global behavior of this complex environment.

One simple way of connecting all these IoT sensors to a central processing unit in an already constructed building is to use wireless communications [1]. Obviously, it's easier to use wireless devices than using a large network of cables throughout the building. But for a large scale building, this wireless connectivity could be harsher to obtain, due to the high pathloss exponent of the radiowaves propagation. Particularly, it is well-known that wireless propagation between multiple floors could be difficult to perform or even to predict efficiently [2].

One way of solving this problem of multi-floor communication in existing buildings is to use Power Line Communications [3]. But in this paper we will focus on reusing another existing network: the ventilation network. Large buildings have usually a centralized ventilation network (known as HVAC, for Heating, Ventilation and Air-Conditioning). This HVAC networks is composed of metallic ducts connecting all rooms to a central fan system.
This network of ducts naturally goes through all floors without obstacles. Therefore it comes that using these ducts as a communication medium to centralize data of remote sensors could be a real breakthrough. A seminal work based on this idea was done by Nikitin [4]. He studied this medium as a cylindrical waveguide in order to evaluate the propagation properties. We reuse there the same principle, then the next section will present a comparison between this waveguide approach, simulations using a 3D EM solver and some measurements. This preliminary study aims at evaluating the practical feasibility of this type of communication depending on the used frequencies and the duct diameters. Then, the third section presents some more realistic measurements, in order to validate the previous study, but also to build a simple empirical model, easy to use to dimension a full network. Finally, the fourth section details the design of a specific antenna for the $2.45 \mathrm{GHz}$ ISM band, with a particular constraint of robustness against changes in the placement of the wireless device in the duct, and in the diameter of this duct.

\section{PROPAGATION IN VENTILATION DUCTS}

\section{A. Propagation Mechanisms}

According to [4], a wireless transmission could be established in a metallic duct, using the waveguide properties of this metallic tube. Considering that this duct

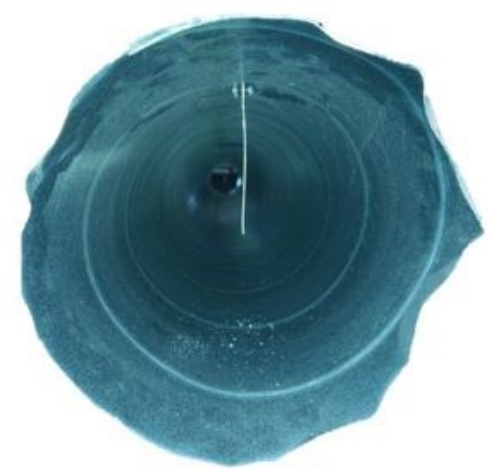

Fig. 1. Quartewavelength monopole antenna inserted in a metallic duct. 


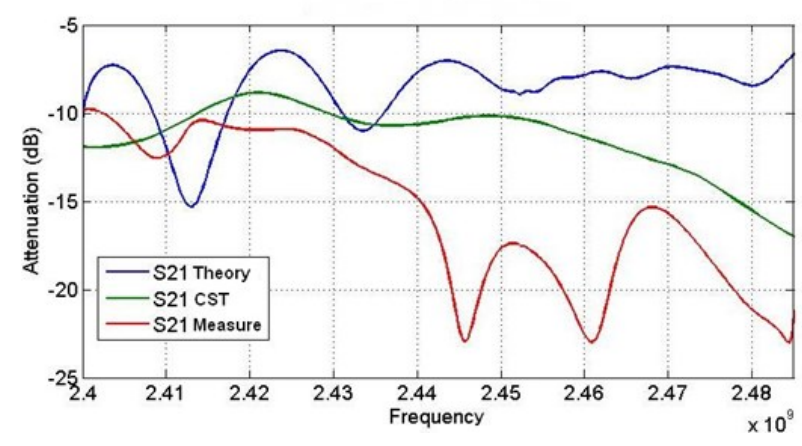

Fig. 2. Comparison of the transmission coefficient $\mathrm{S} 21$ in a metallic duct (diam. 200mm, length $3 \mathrm{~m}$ ) using waveguide theory, EM simulator or measurements.

acts as a cylindrical waveguide, the basic theory (i.e. in [5]) enables to evaluate the number of propagating modes in a cylindrical duct, depending on its diameter and on the used radiowave frequency. [4] has also proposed a complete transfer function of a duct, depending on the previous parameters, the impedance of used antennas at both sides and the length of the duct. Based on this expression, we can theoretically evaluate the attenuation for a certain duct at a certain frequency.

This constitutes a good first step to design such a system, at least because we can from this that, depending on the used frequency, propagation can occur or not in a certain duct. In fact, ducts used in such HVAC systems have normalized diameters (roughly from 85 up to 500 $\mathrm{mm})$.

Considering that the main frequency bands used for IoT systems are at $433 \mathrm{MHz}, 868 \mathrm{MHz}$ or $2.45 \mathrm{GHz}$, we can evaluate the propagation characteristics for each diameter. To this purpose, the waveguide theory already gives us an approximate value of the cutoff frequency for the fundamental mode in a cylindrical waveguide:

$$
f_{c}=1.841 * c /(\pi * d)
$$

For instance, it appears that for a duct of diameter 200 $\mathrm{mm}$, the cutoff frequency is $879 \mathrm{MHz}$, meaning that using 433 or $868 \mathrm{MHz}$ will lead to no propagating modes. Reversely, using $2.45 \mathrm{GHz}$ enables to use diameters down to $72 \mathrm{~mm}$, which is sufficient in all configurations.

\section{B. Propagation Prediction}

To go deeper in that study, we have compared this theoretical model with full-wave simulations and measurements in the following.

This first test was based on a simple straight duct, with a diameter of $200 \mathrm{~mm}$ and a total length of 3 meters. Each end is open, and we used simple quarterwavelength monopole antennas at both sides (see Fig. 1). With this setup, we can compare the theoretical approach (via an implementation on Matlab still based on [4]) with a fullwave simulation of the whole structure (with CST

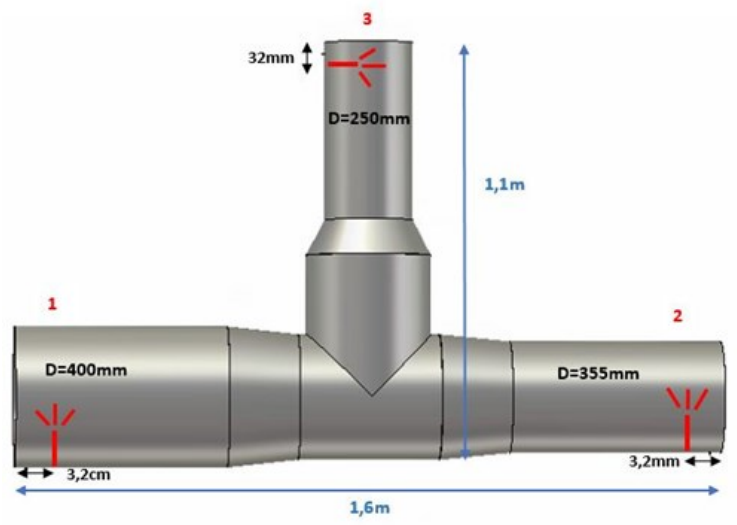

Fig. 3. Example of a complex $\mathrm{T}$ junction with different diameters modeled using CST.

Microwave Studio) and with the measured S21 using a VNA.

It can be seen on Fig. 2 that around $2.45 \mathrm{GHz}$ the global attenuation is quite low, therefore meaning that a transmission could be potentially performed on long distances. We observe also that the theoretical implementation is optimistic, and that the full-wave simulation is closer to the actual measurements.

Apart from these results we also verified that in the same duct, the attenuation at $868 \mathrm{MHz}$ is prohibitive: more than $70 \mathrm{~dB}$ on such a short distance (note that of course we used antennas correctly matched at that frequency).

\section{Complex structures}

In a practical HVAC network, the radiowave signal will encounter much more complex structures than a pure straight duct. Starting from the central fan, the global network globally obey to a tree structure, with very heterogeneous lengths, diameters and directions. Particularly, there are numerous $\mathrm{T}$ junctions, lots of bents and also reducers between different sections. In order to evaluate further the potential link budget of such communications, we have extensively studied the influence of all these specific structures, as well as the influence of antenna placement and polarization. EM simulations (i.e. a T junction like in Fig. 3) have demonstrated that even with such complex structures the global attenuation seems still affordable. For example, a simple bent just add roughly 1.5 $\mathrm{dB}$, where a $\mathrm{T}$ junction could reach up to $8 \mathrm{~dB}$.

\section{FIRST MEASUREMENTS AND EMPIRICAL MODEL}

The main goal of our study is to evaluate the actual potential of such communications in ventilation ducts, but also to provide an easy way to dimension such a network. To this end, after the theoretical and simulation part, we have conducted some first measurements and we have established a very simple empirical model. 


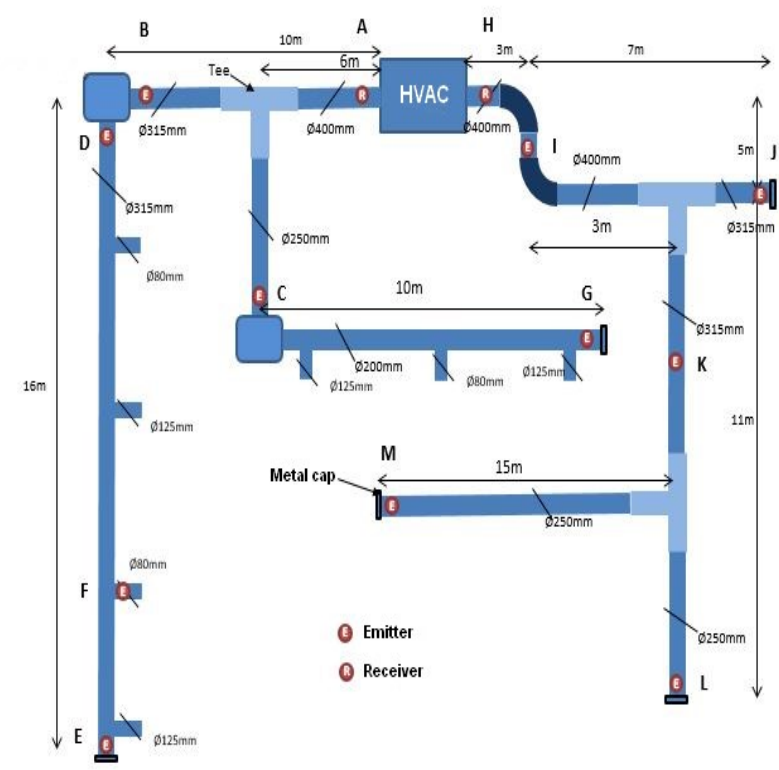

Fig. 4. Detailed scheme with all dimensions of the experimental ventilation network. The source is placed at point $\mathrm{A}$ for measurements at points $B$ to $G$, and at point $H$ for measurements at points I to $\mathrm{M}$.

\section{A. First Measurements}

These first measurements were conducted on a complete network of ventilation that we have constructed on purpose to be representative of most of the actual configurations (see Fig. 4 and 5). This networks includes several branches, with different diameters and with numerous $\mathrm{T}$ junctions and bents.

Two different measurements were performed: one set of measurements using a VNA (vector network analyzer), and another set using ZigBee transceivers (homemade system based on off-the-shelf components, mainly XBee chips and Arduino platforms). For each measurement the source antenna (still a monopole) is placed close to the central engine (points A or $\mathrm{H}$ on Fig. 4). A sample of the measurement results is shown on Table 1, giving the global attenuation of the radio link for both types (VNA and ZigBee based). Globally these two measurements are in good agreement and confirm that even in complex conditions, the attenuation is very low compared to freespace propagation.

TABLE I. MEASUREMENT RESULTS

\begin{tabular}{|c|c|c|c|c|}
\hline Segment & AE & AG & HM & HL \\
\hline $\begin{array}{c}\text { Mean RSSI } \\
\text { (dB) }\end{array}$ & -22 & -30 & -27 & -22 \\
\hline $\begin{array}{c}\text { Mean VNA } \\
\text { (dB) }\end{array}$ & -29 & -33 & -27 & -24 \\
\hline
\end{tabular}

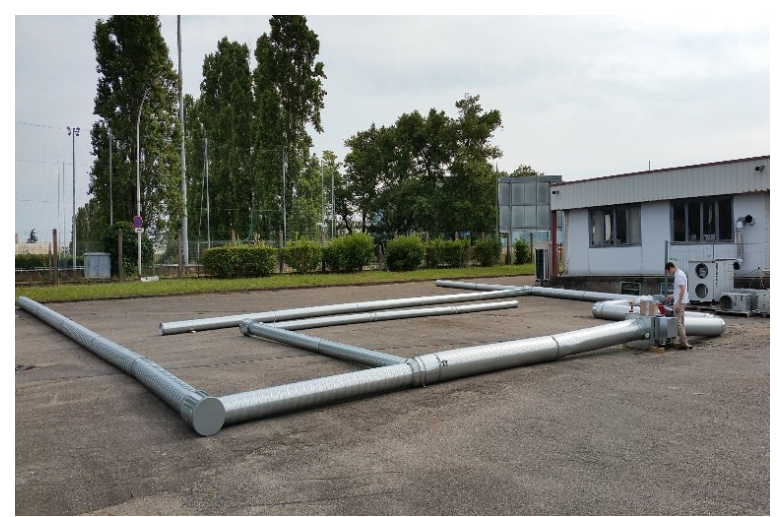

Fig. 5. Picture of the experimental setup.

\section{B. Empirical Model}

Combining these measurement results with an extensive set of simulations, we have built an empirical model, enabling to roughly evaluate the attenuation of a wireless link in this particular medium. This model was constructed for radiowaves at $2.45 \mathrm{GHz}$ and could be expressed as:

$$
\begin{aligned}
& A(d B)=-15.8-L \times 0.1385-K \times 2-M \times 6 \pm 2-C \times \\
& 1.5
\end{aligned}
$$

where $\mathrm{L}$ is the total duct length, $\mathrm{K}$ the number of $\mathrm{T}$ junction in direct connection, $\mathrm{M}$ is the number of $\mathrm{T}$ junctions in orthogonal connection and $\mathrm{C}$ is the number of orthogonal bents.

This expression could be really useful for practical deployment of such systems, but also summarize the main keypoints impacting this waveguide propagation. The first term $(-15.8 \mathrm{~dB})$ corresponds to propagation in a half space (actually $2 \pi$ of steradians). The second point is that we have a linear dependency (in $\mathrm{dB}$ ) to the distance, which is of course much more effective than classical propagation laws in freespace. Globally, the effect of changes in the diameters of the ducts has very little impact on this attenuation, as long as we respect the condition specified in section II.A. on the minimum diameter relative to the frequency.

And finally, only orthogonal $\mathrm{T}$ junctions are really attenuating the signal, but globally a very large duct network could be used without risking an unreachable budget link. For instance, we can use up to 380 meters of duct with $4 \mathrm{~T}$ direct junctions and $4 \mathrm{~T}$ orthogonal junctions to raise the attenuation to more than $100 \mathrm{~dB}$, which is still affordable with common low power wireless standards.

\section{SPECIFIC ANTENNA DESIGN}

After the theoretical study, the extensive simulation set and the first measurements, we have concluded that such a system could really be actually efficient. But one delicate 


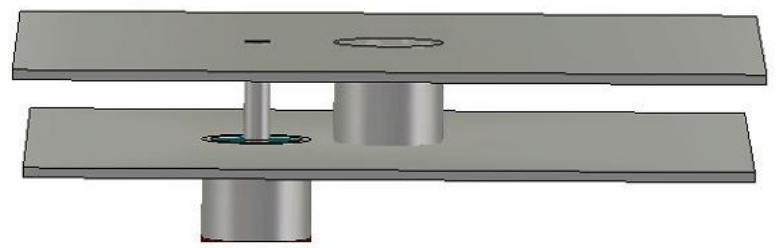

Fig. 6. Global structure of the designed wire-patch antenna optimized for the $2.45 \mathrm{GHz}$ frequency band in a metallic duct.

point remaining is that the antennas located in this metallic surrounding will be very sensitive to their placement and to the diameter of the duct. Moreover, to reduce the impact on the ventilation system, we must privilege the design of a low-profile transceiver.

To this end we have designed and optimize a structure of a wire-patch antenna [6] as presented on Fig. 6. This structure is purely metallic and composed mainly of two parallel square plates, a feeding pin and a shorting pin connected to the ground (lower metallic plate). Le largest dimension is $34 \mathrm{~mm}$ and the height is lower than $5 \mathrm{~mm}$.

The two main advantages of this antenna are that:

- Its radiation pattern is omnidirectional in the horizontal plane, therefore rendering it independent to the placement in terms of radiated gain;

- It is quite robust to the environment and to manufacturing errors.

We have designed this antenna in order that, changing its position in the duct, changing the duct diameter, but also changing the exact values of its dimensions, it always keep a good impedance matching to a 50 ohms basis. For example, Fig. 7 shows that the reflection coefficient remains low enough on the whole band from 2.4 up to 2.5 $\mathrm{GHz}$ when we place the antenna in all possible duct diameters.

This robustness is really a key point to propose an operational system in these specific conditions.

\section{CONCLUSION}

We have presented here a preliminary study of wireless transmissions using the ventilation metallic ducts as waveguides. Starting from the waveguide theory, we have deeply studied in simulation the actual attenuation encountered by radiowaves in such a specific medium. Particularly, T junctions could present an important impact, but still, we have shown in theory, simulation and also in an experimental setup, that this kind of wireless link could achieve a really interesting range in realistic conditions. This paper also expresses a very simple empirical model in order to ease dimensioning a wireless network in such conditions.

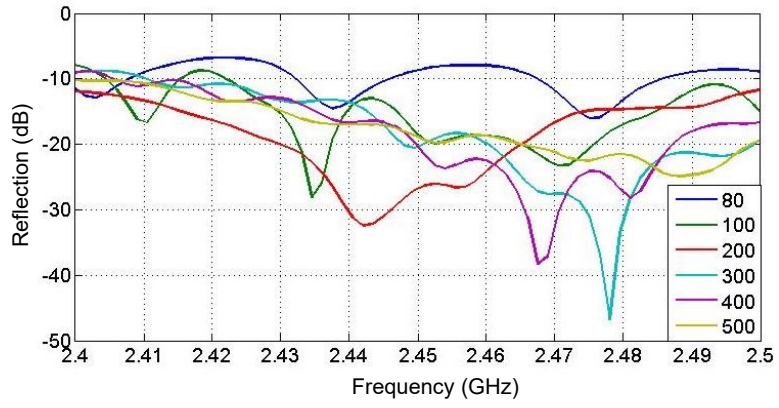

Fig. 7. Reflection coefficient for the proposed antenna depending on the diameter of the metallic duct (in $\mathrm{mm}$ ).

Finally, the antenna design is also a key point to achieve an operational system. We propose here a design of a wirepatch antenna which offers both good performance and high robustness to the influence of the environment.

The future work will be to go to an integrated prototype to test it in real conditions on a long period, thus fully validating this principle, paving the way to commercial products.

\section{ACKNOWLEDGMENT}

This work was supported by a contract and in strong collaboration with the Atlantic Group [7].

\section{REFERENCES}

[1] T. G. Stavropoulos, A. Tsioliaridou, G. Koutitas, D. Vrakas, I Vlahavas, K. Diamantaras, W. Duch, L. S. Iliadis, "System Architecture for a Smart University Building", Artificial Neural Networks, ICANN 2010: 20th International Conference, Thessaloniki, Greece, September 15-18, 2010.

[2] G. De Le Roche, X. Gallon, J.M. Gorce, G. Villemaud, "A 2.5D extension of Frequency Domain ParFlow Method for $802.11 \mathrm{~b} / \mathrm{g}$ propagation simulation in multifloored buildings". IEEE VTC Fall 2006, Montréal, sept 2006.

[3] H. C. Ferreira, H. M. Grové, O. Hooijen, A. J. Han Vinck, "Power Line Communication", ed. John Wiley \& Sons, 2010.

[4] P. V. Nikitin, D. D. Stancil, A. G. Cepni, O. K. Tonguz, A. E. Xhafa and D. Brodtkorb, "Propagation model for the HVAC duct as a communication channel," in IEEE Transactions on Antennas and Propagation, vol. 51, no. 5, pp. 945-951, May 2003.

[5] R. E. Collin, "Field Theory of Guided Waves", 2nd ed., WileyIEEE Press, 1990, 864 pages.

[6] C. Decroze, G. Villemaud, F. Torres and B. Jecko, "Single feed dual mode wire patch antenna," IEEE Antennas and Propagation Society International Symposium, San Antonio, USA, 2002, pp. 22-25 vol.1.

[7] http://www.atlantic.fr/ 